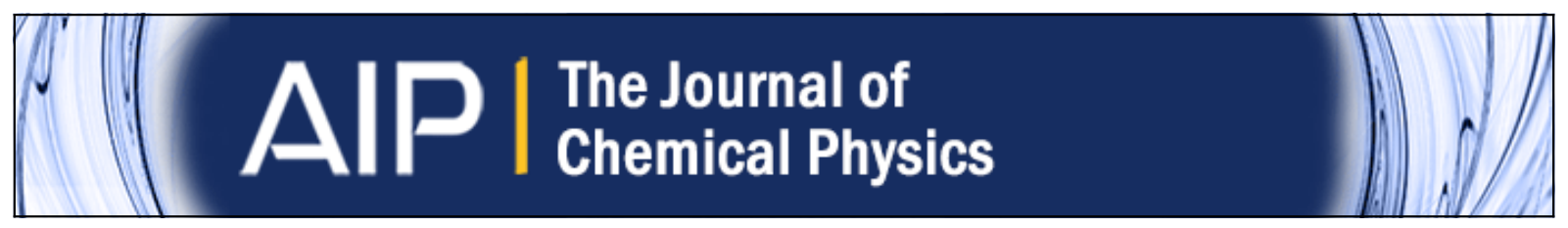

\title{
Effect of compressibility in bubble formation in closed systems
}

K. S. Glavatskiy, D. Reguera, and D. Bedeaux

Citation: The Journal of Chemical Physics 138, 204708 (2013); doi: 10.1063/1.4807323

View online: http://dx.doi.org/10.1063/1.4807323

View Table of Contents: http://scitation.aip.org/content/aip/journal/jcp/138/20?ver=pdfcov

Published by the AIP Publishing

\section{AlP Re-register for Table of Content Alerts}




\title{
Effect of compressibility in bubble formation in closed systems
}

\author{
K. S. Glavatskiy, ${ }^{1}$ D. Reguera, ${ }^{2}$ and D. Bedeaux ${ }^{1}$ \\ ${ }^{1}$ Department of Chemistry, NO 7491, Norwegian University of Science and Technology, Trondheim, Norway \\ ${ }^{2}$ Departament de Física Fonamental, Universitat de Barcelona, Martí i Franquès 1, 08028 Barcelona, Spain
}

(Received 22 February 2013; accepted 3 May 2013; published online 29 May 2013)

\begin{abstract}
We analyze the stability of small bubbles in a closed system with fixed volume, temperature, and number of molecules. We show that there exists a minimum stable size of a bubble. Thus there exists a range of densities where no stable bubbles are allowed and the system has a homogeneous density which is lower than the coexistence density of the liquid. This becomes possible due to the finite liquid compressibility. Capillary analysis within the developed "modified bubble" model illustrates that the existence of the minimum bubble size is associated to the compressibility and it is not possible when the liquid is strictly incompressible. This finding is expected to have very important implications in cavitation and boiling. () 2013 AIP Publishing LLC. [http://dx.doi.org/10.1063/1.4807323]
\end{abstract}

\section{INTRODUCTION}

The formation and stability of bubbles play a very important role in a wide variety of systems of scientific and technological interests. Examples include foaming, cavitation, ${ }^{1}$ sonoluminescence, ${ }^{2}$ or explosive boiling of liquids ${ }^{3}$ that may pose a serious hazard in metallurgic processes, nuclear reactors or cryogenic systems. ${ }^{4-7}$ In most cases, the formation of these bubbles takes place in nucleation phenomena whose intricacies have not been quantitatively understood up to date. Part of the problem lies on the fact that bubbles in open systems are intrinsically unstable, so it is not easy to determine accurately their thermodynamic properties and their kinetic evolution. Moreover, the critical bubble sizes of interest for cavitation and nucleation phenomena are typically very small, ${ }^{8-10}$ and that adds an extra complication to their description. However, it is in principle possible to stabilize bubbles in a closed system, where the volume and total number of molecules (or mass) are fixed.

The stability of small droplets has been discussed in the literature and depends on the thermodynamic control parameters and constraints. ${ }^{11-16}$ In particular one distinguishes between open and closed systems. In open systems, where one controls the chemical potential, the only possible extrema of the grand-potential are maxima that correspond to the critical nuclei extensively studied in the context of nucleation. The formation of small drops in closed systems has also been the subject of studies using different theoretical models, molecular dynamics and Monte Carlo simulations, as well as density functional theory. ${ }^{1-24}$ For closed systems it is the Helmholtz free energy which decides on the stability of the system, and was found to have in general a maximum (i.e., a barrier to nucleation), and a minimum, corresponding to stable equilibrium between the droplet and the surrounding vapor. ${ }^{12-14,16,23,24}$ Even though in open systems there can exist unstable nuclei of arbitrary small size, this is not the case for closed systems, with fixed volume and number of particles. In particular, it was shown earlier, ${ }^{14}$ that in such systems there exist a minimum size of both unstable nuclei and stable droplets due to the undersaturation of the surrounding gas accompanying the formation of a liquid drop.

The analogous problem of the stability of small cavities and bubbles in closed containers has not been so extensively studied, with some notable exceptions. ${ }^{15,23,25,26}$ Moreover, the potential existence of a threshold minimum size and the importance of the fluid compressibility has, to the best of our knowledge, not been analyzed before.

The goal of this paper is to analyze the stability of small bubbles in closed systems. We will see that, surprisingly, it is impossible to stabilize very small bubbles even in closed systems. The underlying reason for that is the finite compressibility of the liquid. Remarkably and contrary to the case of liquid drop formation, where in most cases the effects of compressibility can be safely ignored, the consideration of the compressibility of the liquid is crucial and changes the physics of the problem drastically. In particular, it appears that the different reasons are responsible for the existence of minimum droplet and bubble size in a closed system.

Furthermore, we will see that a simple capillary model can describe very accurately the properties of these small bubbles and their stability, provided that compressibility of the liquid is properly accounted for. For the sake of clarity and simplicity, the calculations will be performed for a simple van der Waals fluid. However, the conclusions and analysis remain valid for more realistic fluids. Square gradient model will be used as a benchmark to describe the thermodynamics of the bubble and to test the accuracy of a simple capillary model which is an adaptation of the "modified liquid drop model" 14,27 to the case of bubble formation.

The paper is organized as follows. In Sec. II we describe the bubble interface with the help of the square gradient model. We show that the density profile obtained from the square gradient model becomes flat if the total mass of the system exceeds a threshold mass, indicating that very small bubbles are not stable in the system. Next, in Sec. III we consider a simple capillary model for a bubble. Its solution for the bubble size dependence on the mass has a locally stable and unstable branches. The locally stable branch of this model coincides with the results of the square gradient model. Analysis 
of the capillary model allows us to understand the reason for the existence of the minimum bubble size. Finally, in Sec. IV we give concluding remarks.

\section{SQUARE GRADIENT MODEL}

We first describe the system using the square gradient model (SG), ${ }^{28,29}$ the simplest approximate form of density functional theory. ${ }^{30-33}$ Within this model the local Helmholtz energy density $f(\mathbf{r})$ depends not only on the local mass density $\rho(\mathbf{r})$ but also on the derivatives of the density with respect to the position. This dependence is important in the interfacial region where the density changes abruptly over a very small distance. Typically, it is sufficient to consider the first term in the Taylor expansion of the Helmholtz energy in the density derivatives, so that for a one-component system the Helmholtz energy density takes the following form: ${ }^{34}$

$$
f(\mathbf{r})=f_{0}(\rho(\mathbf{r}), T)+\frac{1}{2} \kappa(\rho(\mathbf{r}))|\nabla \rho(\mathbf{r})|^{2}
$$

where $f_{0}(\rho(\mathbf{r}), T)$ is the bulk Helmholtz energy and $\kappa$ is a parameter related to the surface tension which may depend on the density. The equilibrium state in a closed system is characterized by a solution profile $\rho(\mathbf{r})$ that minimizes the total Helmholtz energy $F[\rho(\mathbf{r})]=\int d \mathbf{r} f(\mathbf{r})$ with the constraint that the total mass (or number of molecules) $m_{\text {tot }}=\int d \mathbf{r} \rho(\mathbf{r})$ is fixed. This requires the minimization with respect to the density of the functional,

$$
\int d \mathbf{r} f(\mathbf{r})-\mu_{e} \int d \mathbf{r} \rho(\mathbf{r}),
$$

where $\mu_{e}$ is the Lagrange multiplier which is equal to the equilibrium chemical potential. Thus the actual density profile can be found by solving the equation,

$$
\mu_{e}=\mu_{0}(\rho, T)-\frac{1}{2} \frac{\partial \kappa}{\partial \rho}|\nabla \rho(\mathbf{r})|^{2}-\kappa \Delta \rho,
$$

where $\mu_{0}(\rho, T) \equiv \partial\left(\rho f_{0}(\rho, T)\right) /(\partial \rho)$ and $\Delta \equiv \nabla \cdot \nabla$ is the Laplace operator. This is the profile which minimizes the Helmholtz energy for given conditions.

In spherically symmetric system where all the quantities depend only on the radial coordinate this equation takes the following form:

$$
\mu_{e}=\mu_{0}(\rho, T)-\frac{1}{2} \frac{\partial \kappa}{\partial \rho} \rho^{\prime 2}-\kappa\left(\rho^{\prime \prime}+\frac{2}{r} \rho^{\prime}\right),
$$

where prime indicates derivative with respect to the radius.

The chemical potential $\mu_{0}(\rho, T)$ can be found from the bulk equation of state. We use van der Waals equation of state

$$
p_{0}(v, T)=\frac{R T}{v-B}-\frac{A}{v^{2}},
$$

where $v=M / \rho$ is the molar volume and $M$ is the molar mass, while $A$ and $B$ are the van der Waals coefficients. We refer to Refs. 34, 35 for the details of the calculations using this equation of state. For the chemical potential this gives

$$
\mu_{0}(v, T)=R T\left(\frac{B}{v-B}-\ln \frac{v-B}{N_{a} \Lambda^{3}}\right)-2 \frac{A}{v},
$$

where $\Lambda$ is the mean de Broglie wavelength, which depends on the temperature but not on the molar volume, and that appears since the chemical potential of an ideal gas has been taken as a reference.

The second order differential equation, Eq. (4), requires two boundary conditions. For the equilibrium profile these conditions should be the following: $\rho^{\prime}(0)=0$ and $\rho^{\prime}(L)=0$, where $L$ is the radius of the spherical container. The former one reflects the smoothness of the density profile at the origin and prevents singular solutions, while the latter one reflects the fact that we have the bulk fluid phase at the outer boundary. The advantage of this choice is that, on the one hand, there is no influence of the nature of the wall on the calculation, and on the other hand, it facilitates a closer comparison with the properties of an equivalent bubble in an open system, since it mimics a bulk system. This choice of zero gradient at the wall has also been used in previous density functional theory studies of small drops in the canonical ensemble (i.e., a closed system). ${ }^{24}$ We note, that even if choosing zero gradient at the wall is a natural condition to impose for open system, the system is still closed because of the extra condition of fixing the total mass in the system.

In a closed system, where one controls the total mass $m_{\text {tot }}$ (rather than the pressure or the chemical potential), the equilibrium chemical potential $\mu_{e}$ is not imposed but has to be obtained instead from the differential equation. For this we introduce the mass of the system $m(r)$ as an additional variable, which satisfies the equation $m^{\prime}(r)=4 \pi r^{2} \rho(r)$ and gives the two additional boundary conditions for the mass: $m(0)=0$ and $m(L)=m_{\text {tot }}$.

Thus we have three variables $m(r), \rho(r)$ and $\rho^{\prime}(r)$ and one unknown parameter $\mu_{e}$. Solving the above differential equations together with the given boundary conditions we can obtain the density profile across the system.

The typical profile obtained from the square gradient theory shows that there exists a region of a finite width where the density changes continuously from the gas-phase value to the liquid-phase value, the interfacial region. One has therefore a freedom to define the size of the bubble anywhere within this region. A typical choice is to introduce the equimolar dividing surface $R_{\rho}$, such that

$$
0=\int_{0}^{L}\left[\rho(r)-\rho^{g} \Theta\left(R_{\rho}-r\right)-\rho^{\ell} \Theta\left(r-R_{\rho}\right)\right] 4 \pi r^{2} d r,
$$

where $\Theta(x)$ is the step function, while $\rho^{g}$ and $\rho^{\ell}$ are the extrapolated (constant) values of the gas and liquid density. The radius of the bubble is therefore defined as $R_{\rho}$ and can be obtained from the solution of the square gradient model as a function of the total mass in the system.

For illustrative purposes, we will particularize the calculations for the case of cyclohexane whose relevant thermophysical properties are listed in Table I.

At temperature $T=330 \mathrm{~K}$ this gives a value for the coexistence pressure and chemical potential at planar interface of $p_{\infty}=340550 \mathrm{~Pa}$ and $\mu_{\infty}=29744 \mathrm{~J} / \mathrm{mol}$ (with an ideal gas as a reference), respectively. For the calculations, we will use a spherical container with radius $L=80 \mathrm{~nm}$. The mass of 
TABLE I. Material properties of cyclohexane used.

\begin{tabular}{lcc}
\hline \hline$M$ & 0.084162 & $(\mathrm{~kg} / \mathrm{mol})$ \\
$A$ & 2.195 & $(\mathrm{~J} \mathrm{~m} / \mathrm{mol})$ \\
$B$ & $14.13 \times 10^{-5}$ & $\left(\mathrm{~m}^{3} / \mathrm{mol}\right)$ \\
$\sigma$ & 0.0370 & $(\mathrm{~N} / \mathrm{m})$ \\
$\kappa$ & $1.7282 \times 10^{-15}$ & $\left(\mathrm{~J} \mathrm{~m}^{5} / \mathrm{kg}^{2}\right)$ \\
\hline
\end{tabular}

the system will be varied between $m_{g}=2.4604 \times 10^{-20} \mathrm{~kg}$ $=1.4817 \times 10^{7}$ a.m.u., when only gas at coexistence density $\rho_{g, \infty}=11.4724 \mathrm{~kg} / \mathrm{m}^{3}$ is present in the box, to $m_{\ell}=9.8690$ $\times 10^{-19} \mathrm{~kg}=5.9432 \times 10^{8}$ a.m.u., when only liquid at coexistence density $\rho_{\ell, \infty}=460.164 \mathrm{~kg} / \mathrm{m}^{3}$ is present in the box.

We have analyzed the behavior of the bubble when the total mass of the system changes using the square gradient (SG) theory together with the van der Waals equation of state. Typical density profiles are given in Fig. 1. The profiles were obtained with the help of MATLAB procedure bvp4c with a relative accuracy $10^{-9}$. After several trial profiles the numerical solution converged to the one presented in the figure. All of the profiles correspond to the minima in the Helmholtz free energy.

One can see from Fig. 1 that density changes from the gas value to the liquid value. The density profile becomes flat when the total mass exceeds a threshold value. We also plot the dependence of the equimolar radius of the bubble $R_{\rho}$ as a function of the total mass $m_{\text {tot }}$ in Fig. 2. As expected, the size of the bubble decreases when the total mass increases. However, surprisingly there exist a certain mass threshold $m_{b}$ $=9.4567 \times 10^{-19} \mathrm{~kg}=5.6950 \times 10^{8}$ a.m.u., which is less than $m_{\ell}$ after which the bubble does not exist. This is indicated in Fig. 2 by the jump in the $R_{\rho}\left(m_{t o t}\right)$ dependence obtained from the continuous profile. When $m_{\text {tot }}<m_{b}$ bubble size remains finite, reaching a minimum value $R_{\text {min }}=17.7$ $\mathrm{nm}$ at $m=m_{b}$. When $m_{\text {tot }}>m_{b}$, the size of the bubble jumps to zero and remains zero for all masses in the interval $\left(m_{b}, m_{\ell}\right)$, indicating that the equilibrium state in that case corresponds to a homogeneous liquid filling the whole box. Thus it is clear that it is impossible to have a stable bubble of

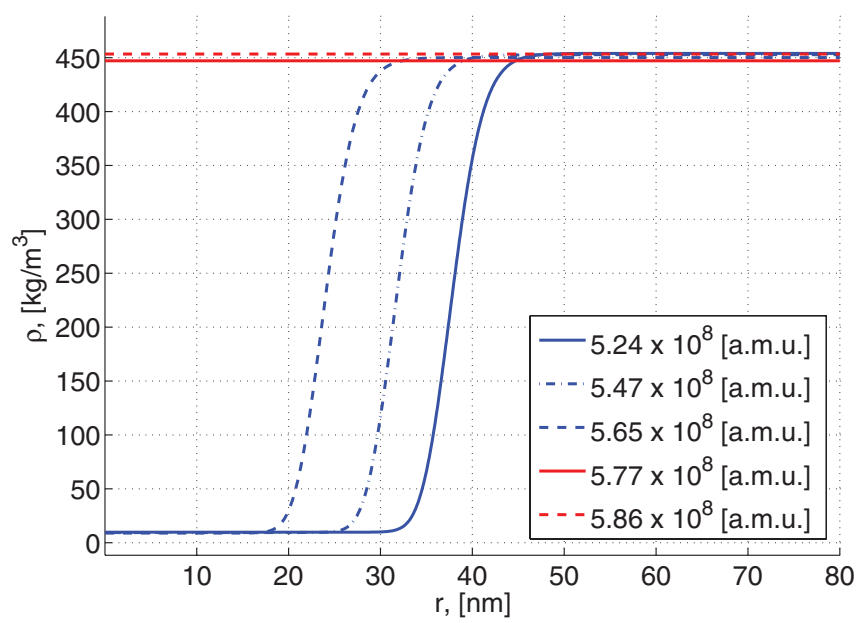

FIG. 1. Density profile obtained from the square gradient model for different total mass of the system. radius smaller of $R_{\min }$ in a closed container. Notice that in our particular example, this minimum size turns out to be quite large (i.e., $17.7 \mathrm{~nm}$ ) compared to the typical critical bubble sizes which trigger cavitation and boiling.

\section{MODIFIED BUBBLE MODEL}

In order to understand this puzzling result, we will introduce a simple capillary model which is developed in analogy with the "modified liquid drop model", 14,27 and accordingly will be called the "modified bubble model." The model, illustrated in Fig. 3, consists of a spherical container of fixed radius $L$, containing $N$ molecules and a total mass $m_{\text {tot }}$ at temperature $T$. At low enough total mass, a bubble can form inside the spherical container. The bubble is considered to have a sharp interface of radius $R_{\sigma}$, a volume $V_{b}=4 / 3 \pi R_{\sigma}^{3}$ and contains $n$ gas molecules. The liquid then occupies the remaining volume $4 / 3 \pi\left(L^{3}-R_{\sigma}^{3}\right)$ and is made of the remaining $N-n$ molecules. Thus, for a given total mass $m_{\text {tot }}$ and bubble radius $R_{\sigma}$, the densities of the gas and the liquid are $\rho_{g}=m_{1} n / V_{b}$ and $\rho_{\ell}=m_{1}(N-n) /\left(V-V_{b}\right)$, respectively, where $m_{1}=m_{t o t} / N$ is the molecular mass of the fluid. Following the derivation of the "modified liquid drop model," it is straightforward to show that the equilibrium conditions for a bubble of radius $R_{\sigma}$ are given by the standard equations,

$$
\begin{aligned}
\mu_{g}\left(p_{g}, T\right) & =\mu_{\ell}\left(p_{\ell}, T\right) \\
p_{\ell}-p_{g} & =-\frac{2 \sigma}{R_{\sigma}},
\end{aligned},
$$

where $\sigma$ is the surface tension of the bulk planar fluid, $\mu_{g}$ and $\mu_{\ell}$ are the chemical potentials of the gas and liquid phase, while $p_{g}$ and $p_{\ell}$ are the corresponding pressures. The chemical potentials of each phase can be calculated from the GibbsDuhem equation at constant temperature, with the assumption that the surface tension is independent of pressure (cf. also Ref. 14):

$$
\begin{aligned}
\mu & =\mu_{\infty}\left(p_{\infty}\right)+\int_{p_{\infty}}^{p} v(p) d p \\
& =\mu_{\infty}\left(p_{\infty}\right)+\int_{v_{\infty}}^{v} v \frac{\partial p}{\partial v} d v
\end{aligned}
$$

where $v$ is the molar volume, while $p_{\infty}$ and $\mu_{\infty}$ are the coexistence pressure and chemical potential at planar interface.

Within the capillary approach one can consider different models of the bulk phase. We will consider the following: (i) the standard approximation made in the classical theory, where the bubble is considered as an ideal gas surrounded by an incompressible liquid; (ii) a more realistic case, where both gas and liquid are described by the vdW equation of state. In each case, solving Eq. (8) together with Eq. (9) will give the dependence of the bubble size $R_{\sigma}$ on the total mass $m_{t o t}$ of the system.

For the simplest case of a bubble of ideal gas surrounded by incompressible fluid, the chemical potentials can be easily obtained and the equilibrium conditions prescribed by Eq. (8) 


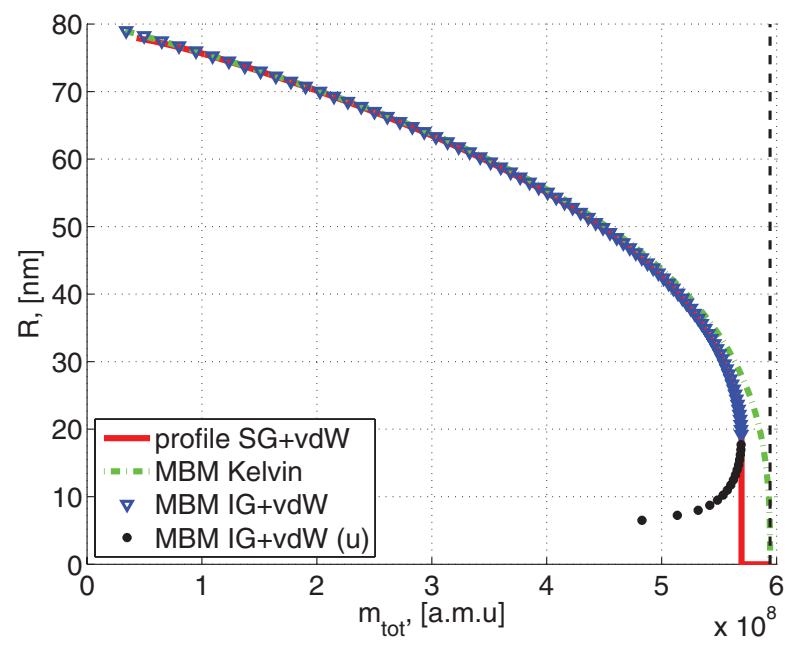

(a)

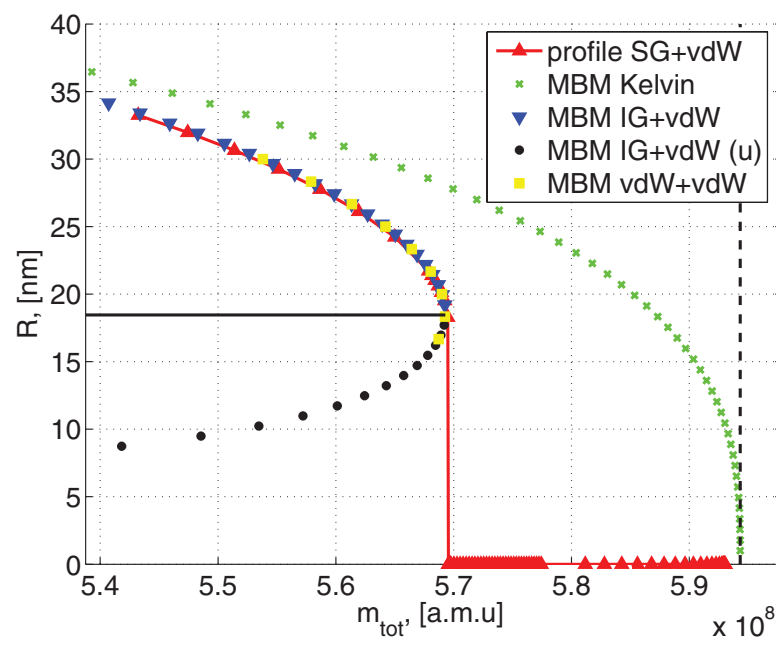

(b)

FIG. 2. (a) Size of the bubble as a function of the total mass obtained from: SG theory ("profile SG+vdW," solid red line); MBM assuming ideal gas and incompressible liquid ("MBM Kelvin," dotted line); MBM with ideal gas and vdW liquid ("MBM IG+vdW," triangles). The unstable branch of "MBM" solution is indicated with black dots. (b) Zoom of the region where the stable bubble disappears. The dashed vertical line indicates value of the mass corresponding to the equilibrium density of the liquid. The $R$ in the caption stands for $R_{\rho}$ and $R_{\sigma}$ in case of the results from SG theory and MBM, respectively.

will then take the following form:

$$
R T \ln \frac{p_{g}}{p_{\infty}}=-\frac{2 \sigma v_{\ell}}{R_{\sigma}}+v_{\ell}\left(p_{g}-p_{\infty}\right),
$$

where $v_{\ell}$ is the molar volume of the incompressible liquid. The last term on the right-hand side is typically very small compared to the contribution of the Laplace pressure and can be safely neglected, leading to the well-known Kelvin equation for the pressure of the vapor phase. This equation has only one solution, corresponding to a stable bubble forming inside the container. Using the value of the surface tension listed in Table I (which, to make a fair comparison, is the one obtained from the square-gradient model with the given $\kappa$ rather than the experimental value), the results of this model are shown by the dotted line also in Fig. 2 together with the results of the SG model. For low masses, the predictions agree with the results of the SG model. However, at large $m_{t o t}$ the results deviate and in this case bubbles of any size are allowed to exist. The profile $R(m)$ goes monotonically to zero when the mass goes to $m_{\ell}$, as can be seen in the zoomed picture shown in Fig. 2(b). Thus, considering the liquid as incompressible,

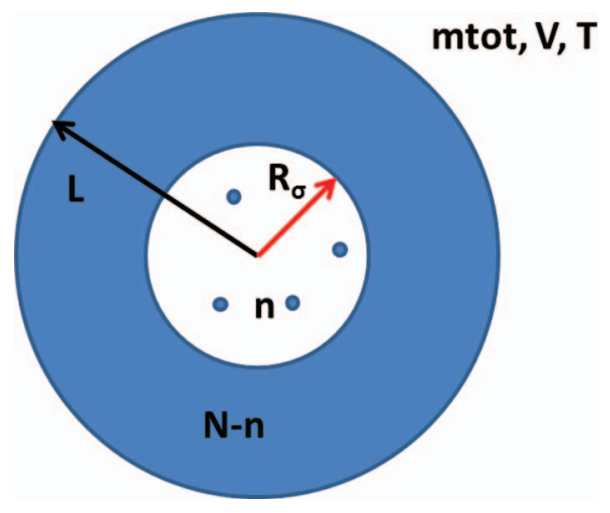

FIG. 3. Schematic illustration of the "modified bubble model" (MBM). which is the standard assumption in the classical theory of nucleation and bubble formation, there is no minimum stable size and one cannot explain the SG results.

However, there is nothing in the capillary approximation that prevents us from using the vdW equation of state to describe the bulk properties of both phases. Thus, in this second model, that we will call MBM, the compressibility of the liquid phase is accounted for. In this case, the chemical potentials $\mu_{g}$ and $\mu_{\ell}$ are found from (6), while the pressures $p_{g}$ and $p_{\ell}$ are found from the vdW equation of state (5). Remarkably, now the equilibrium conditions (8) yield two possible solutions for the radius of the bubble, which are plotted in Fig. 2. The upper branch corresponds to a locally stable bubble, and amazingly matches perfectly the results of the SG model. In fact, it also predicts that there is no solution (i.e., no stable or metastable bubble size) for masses exceeding $m_{b}$. Here, the stability of the bubble is characterized from the Helmholtz energy, using the same analysis as in Ref. 14. In particular, for $m_{\text {tot }}<m_{b}$ the upper branch corresponds to a local minimum of the Helmholtz energy, and thus to a stable or metastable solution, while the lower branch corresponds to a local maximum of the Helmholtz free energy, and thus to an unstable solution. For $m_{t o t}>m_{b}$ the Helmholtz free energy has no local extremum for any bubble size and its lowest value corresponds to a homogeneous liquid filling the whole container.

Both the predictions of the minimum stable bubble size $R_{\min }$ and of the mass (or total density) of the system at which this occurs $m_{b}$, agree perfectly with the results of the SG theory. Figure 2(b) shows a zoomed picture of the results near $m_{b}$ and also plots an extra line corresponding to the solution of the capillary model when the liquid phase is described by the vdW equation, but the gas is treated as ideal. The results are identical to those of MBM, showing that compressibility of the liquid, rather than the negligible nonideality of the gas phase, is the key to understand the impossibility to form small stable bubbles. The lower branch, which is the second 


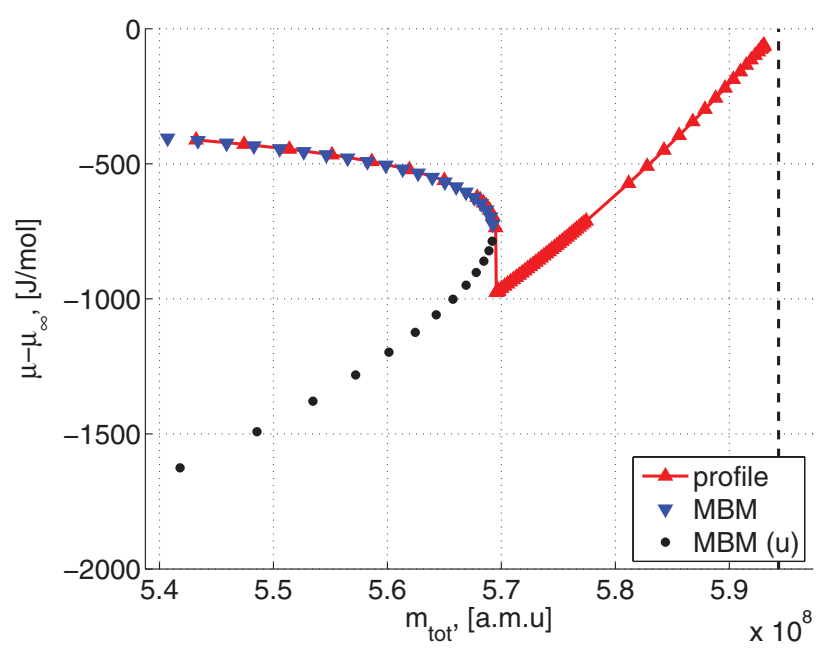

(a)

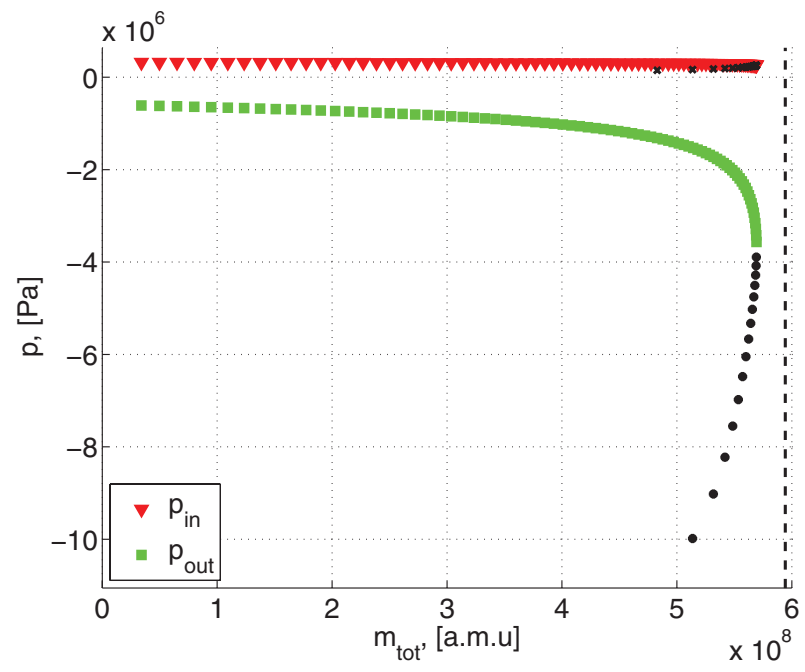

(b)

FIG. 4. (a) Chemical potential excess of the system as a function of the total mass from SG profile and from MBM. Unstable part of MBM solution is indicated by black dots. (b) Pressure inside the bubble and the surrounding liquid as a function of the total mass from MBM. Unstable part of MBM solution is indicated by black dots and crosses. Dashed vertical line indicates the value of the mass corresponding to the equilibrium density of the liquid.

solution of the MBM, for which $R_{\sigma}<R_{\text {min }}$ corresponds to an unstable bubble, a maximum of the free energy landscape of bubble formation (i.e., it is the equivalent to the critical bubble size for an open system). This branch also ends at $m_{b}$ and $R_{\text {min }}$, confirming that larger masses cannot sustain bubbles.

We note, that the effect is observed not only at given temperature of $330 \mathrm{~K}$. The calculations show that the threshold mass $m_{b}$ is always lower than the total possible mass $m_{\ell}$ in the entire range of temperatures from the triple point far enough from the critical point. This implies that the existence of the minimum stable bubble size is independent of the temperature.

It is interesting to see the behavior of the chemical potential of the system close to $m_{b}$, which is shown in Fig. 4(a). It is consistent with the above results. In particular, the mass dependence of the chemical potential from MBM reveals two branches, with only the upper branch being stable, while the lower branch corresponds to an unstable solution. Furthermore, the mass dependence of the chemical potential from SG model reveals the jump at $m_{b}$.

An important clue on the role of compressibility is provided by the plots in Fig. 4(b) of the pressure in the bubble and in the liquid phase as a function of the total mass. Once again we note that the predictions of the MBM perfectly agree with the $\mathrm{SG}$ results. As the radius of the bubble becomes smaller, the Laplace contribution (i.e., the right-hand side of Eq. (8) for the pressure difference) generates a very large negative pressure in the liquid, that stretches the liquid phase and reduces its density compared to the coexistence one. Eventually, when the bubble is very small, the stretching becomes so large, that the liquid phase will fill the entire container collapsing the bubble. Thus, at those conditions, it is energetically more favorable for the system to fill the entire volume with an homogeneous stretched liquid phase rather than to pay the extra interfacial cost and have the large negative pressures in the liquid phase required to accommodate a small bubble. That is the ultimate physical reason why very small bubbles of real compressible fluids cannot be stable in closed containers. In a strictly incompressible liquid, the large tensile pressures associated to the Laplace pressure will not stretch the liquid, which occupies a fixed fraction of the box volume, thus always leaving room for arbitrarily small bubbles.

Another surprising consequence of Laplace's law is the fact the effective compressibility of the system is negative, as evidenced by Fig. 4(b). That might seem a violation of the well-known thermodynamic stability requirement of having a positive compressibility. However, this requirement only applies for a uniform single phase system, and in our case the system contains two phases (liquid and vapor), each of them having a positive compressibility. The effective negative compressibility is not a signature of instability in this case, but rather a natural consequence of the equilibrium between the two phases in a finite system. As we add more molecules to the system, they will condense into the liquid phase, reducing the radius of the bubble. Following Laplace's law, Eq. (8), the only way to keep the vapor pressure near saturation and, at the same time, have this smaller bubble stabilized inside the fixed volume container is by reducing the pressure of the liquid phase. Thus, the pressure of the liquid phase for the system containing this bubble must decrease as the total mass is increased, leading to this effective negative compressibility. The same behavior has been also reported and explained for the inverse situation of drop formation in a closed container in Ref. 14.

\section{CONCLUSIONS}

In this article we have shown that in a closed system one cannot observe very small stable bubbles. Remarkably, the existence and the value of the minimum bubble size one can observe can be obtained accurately from a simple capillary model of the bubble. We emphasize, that in order to observe 
this effect it is crucial to account for the compressibility of the liquid. Incompressible liquids allow bubbles of arbitrarily small sizes. This conclusion is important since compressibility is considered a small effect that is commonly neglected. In fact, compressibility is not important in the inverse situation, i.e., in the formation of liquid drops in supersaturated vapors. In that case, the small change in volume associated to the Laplace pressure changes negligibly the equilibrium with the vapor and the physics of the problem. However, we have seen that compressibility is crucial for the correct description of small bubbles, since in this case the large negative pressures that would be required to stabilize the bubble stretch the liquid phase significantly and make impossible its formation. That leads to the existence of a minimum stable bubble size in closed systems having important implications in cavitation and boiling that will be explored in a future work.

\section{ACKNOWLEDGMENTS}

This work has been supported by VISTA Grant No. 6343 and the MICINN of the Spanish government through Grant Nos. FIS2008-01299 and FIS2011-22603. We acknowledge E. M. Blokhuis for fruitful discussions.

${ }^{1}$ M. El Mekki Azouzi, C. Ramboz, J.-F. Lenain, and F. Caupin, Nat. Phys. 9, 38-41 (2012).

${ }^{2}$ D. J. Flannigan and K. S. Suslick, Nature (London) 434, 52-55 (2005).

${ }^{3}$ V. E. Vinogradov, P. A. Pavlov, and V. G. Baidakov, J. Chem. Phys. 128, 234508 (2008).

${ }^{4}$ M. Blander and J. L. Katz, AIChE J. 21, 833-848 (1975).

${ }^{5}$ R. Cole, Adv. Heat Transfer 10, 85-166 (1974).

${ }^{6}$ V. P. Skripov, Metastable Liquids (John Wiley \& Sons, New York, 1974).
${ }^{7}$ P. G. Debenedetti, Metastable Liquids (Princeton University Press, Princeton, NJ, 1996).

${ }^{8}$ K. Davitt, A. Arvengas, and F. Caupin, EPL 90, 16002 (2010).

${ }^{9}$ Z.-J. Wang, C. Valeriani, and D. Frenkel, J. Phys. Chem. B 113, 3776-3784 (2009).

${ }^{10}$ C. F. Delale, J. Hruby, and F. Marsik, J. Chem. Phys. 118, 792-806 (2003).

${ }^{11}$ A. J. Yang, J. Chem. Phys. 79, 6289 (1983).

${ }^{12}$ A. J. Yang, J. Chem. Phys. 82, 2082 (1985).

${ }^{13}$ D. J. Lee, M. M. Telo da Gama, and K. E. Gubbins, J. Chem. Phys. 85, 490 (1986).

${ }^{14}$ D. Reguera, R. E. Bowles, Y. Djikaev, and H. Reiss, J. Chem. Phys. 118, 340-353 (2003).

${ }^{15}$ L. G. MacDowell, V. K. Shen, and J. R. Errington, J. Chem. Phys. 125, 034705 (2006).

${ }^{16}$ M. P. Moody and P. Attard, J. Chem. Phys. 117, 6705 (2002).

${ }^{17}$ A. E. van Giessen and E. M. Blokhuis, J. Chem. Phys. 131, 164705 (2009).

${ }^{18}$ K. Binder and M. H. Kalos, J. Stat. Phys. 22, 363 (1980).

${ }^{19}$ N. G. Garcia and J. M. S. Torroja, Phys. Rev. Lett. 47, 186 (1981).

${ }^{20}$ J. K. Lee, J. A. Barker, and F. F. Abraham, J. Chem. Phys. 58, 3166 (1973).

${ }^{21}$ F. F. Abraham, J. K. Lee, and J. A. Barker, J. Chem. Phys. 60, 246 (1974).

${ }^{22}$ M. Schrader, P. Virnau, and K. Binder, Phys. Rev. E 79, 061104 (2009).

${ }^{23}$ M. Rao, B. J. Berne, and M. H. Kalos, J. Chem. Phys. 68, 1325 (1978).

${ }^{24}$ V. Talanquer and D. W. Oxtoby, J. Chem. Phys. 100, 5190 (1994).

${ }^{25}$ J. F. Lutsko, EPL 83, 46007 (2008).

${ }^{26}$ M. Uline and D. Corti, Phys. Rev. Lett. 99, 076102 (2007).

${ }^{27}$ C. L. Weakliem and H. Reiss, J. Chem. Phys. 99, 5374-5383 (1993).

${ }^{28}$ J. D. van der Waals, Verh.-K. Ned. Akad. Wet., Afd. Natuurkd., Eerste Reeks 1(8), 56 (1893).

${ }^{29}$ J. W. Cahn and J. E. Hilliard, J. Chem. Phys. 28, 258-267 (1958).

${ }^{30}$ J.-S. Li and G. Wilemski, J. Chem. Phys. 118, 2845-2852 (2003).

${ }^{31}$ D. W. Oxtoby and R. Evans, J. Chem. Phys. 89, 7521-7530 (1988).

${ }^{32}$ D. A. Kashchiev and D. W. J. Oxtoby, J. Chem. Phys. 100, 7665-7671 (1994).

${ }^{33}$ V. K. Shen and P. G. Debenedetti, J. Chem. Phys. 114, 4149-4159 (2001).

${ }^{34}$ K. S. Glavatskiy and D. Bedeaux, Phys. Rev. E 77, 061101-1-061101-15 (2008).

${ }^{35}$ K. S. Glavatskiy, Multi-Component Interfacial Transport as Described by the Square Gradient Model: Evaporation and Condensation, Springer Thesis Book Series (Springer, Berlin, 2011). 